\title{
Rational Approach towards COVID-19 Main Protease Inhibitor (Fexofenadine) Via Molecular Docking
}

\author{
Dr. Bhagwan Nautiyal ${ }^{1}$, Amit Kumar ${ }^{2}$, Jitender K Malik ${ }^{2 *}$ \\ ${ }^{1}$ Smt. Manjira Devi Ayurvedic Medical College \& Hospital, Hitanu, Uttarkashi, India \\ ${ }^{2}$ Smt. Manjira Devi Shikshan and Prashikshan Institute, Hitanu Dhanari, Uttarkashi, India
}

DOI: $10.36348 /$ sjmps.2020.v06i11.008

| Received: 08.11.2020 | Accepted: 19.11.2020 | Published: 30.11.2020

*Corresponding author: Jitender K Malik

\section{Abstract}

An outburst of pneumonia in December, 2019 in Wuhan, China, has now been gritty to be caused by a novel corona virus. Severe Acute Respiratory Syndrome Corona-virus (SARS-CoV) named is allotted for this novel threads. The disease has spread more than 185 countries and regions, with more than 2.05 million confirmed cases and more than 1,34,354 deaths as of April 16, 2020. The pandemic is enduring to spread inspite of worldwide efforts. The SS-RNA virus from the enveloped corona virus family caused SARS (Severe acute respiratory syndrome) which is life threatening viral infection.The spreading of infection is quick in many countries of the world. The World Health Organization (WHO) called COVID-19 a pandemic on March 11, 2020. There are numerous drug trials going on with some positive results. Though, since no vaccine is available, the best way to fight the virus is by preventive measures. In the present research an attempt had been made to find new COVID-19 main protease inhibitor by molecular docking approach. Fexofenadine is an antihistamine that is used to treat the symptoms of seasonal allergies (hay fever) in adults and children. Fexofenadine is also used to treat skin itching and hives caused by a condition called chronic idiopathic urticaria in adults and children at least 6 years old. Fexofenadine was taken as drug which follows Lippinski's rule of five, thus having very good drug score as well as drug likeness score. The present study reveals that Doxycycline has good binding affinity with COVID-19 protease and thus can be used as prophylaxis and therapeutic treatment for corona patient.

Keywords: COVID-19, Fexofenadine, Molecular Docking \& Prevention measures.

Copyright () 2020 The Author(s): This is an open-access article distributed under the terms of the Creative Commons Attribution 4.0 International License (CC BY-NC 4.0) which permits unrestricted use, distribution, and reproduction in any medium for non-commercial use provided the original author and source are credited.

\section{INTRODUCTION}

The earth is peaceful but humans are vanishing. The world experienced coronavirus for the first time in 2002-2003 through severe acute respiratory syndrome (SARS), and in 2011, Middle East respiratory syndrome (MERS) for the first time. The causative agents for both cases (SARS-CoV and MERS-CoV,) were newly identified coronaviruses of zoonotic origin in the genus Beta coronavirus [1]. The COVID-19 pandemic due to SARS-CoV-2 infection can produce Acute Respiratory Distress Syndrome as a consequence of a pulmonary cytokine storm. Antihistamines are secure and useful treatments for reducing inflammation and cytokine release. Combinations of Histamine- 1 and Histamine-2 receptor antagonists have been effective in urticaria, and might reduce the histamine-mediated pulmonary cytokine storm in COVID-19[2]. The body contains the organs of the immune system, which protects against diseases. It plays a key role to maintain health and pathogenesis. It also protects the body from harmful substances, germs, and cell changes (neoplasm). The key player in the immune system is the white blood cells, which can travel throughout the body through the blood vessels. To monitor for invading microbes, the body exchanges cells and fluids between blood and lymphatic vessels and enables the lymphatic system [3]. Histamine and mast cells play a primary role in modulate inflammation through increased capillary blood flow and vascular permeability, as well as cytokine release. Histamine-1 (H1) receptor antagonists (e.g., cetirizine, fexofenadine) are administered for allergies. Histamine-2 (H2) receptor antagonists (e.g., famotidine) are used to control acid in the stomach and heart burn. Prescription branded, generic, and over-the-counter (OTC) drugs of both classes are safe and commercially available worldwide. The disease can manifest as a hyperimmune response with pulmonary cytokine release resembling that of other respiratory infections, such as Severe Acute Respiratory Syndrome (SARS), Middle 
East Respiratory Syndrome (MERS), and influenza. Studies from China have defined the COVID-19 cytokine profile and identified risk factors that increase mortality. These retrospective studies suggest mortality may be linked to inflammatory processes caused by a "cytokine storm", which was very common in patients with severe to critical symptoms. Pulmonary pathology in early-phase COVID-19 pneumonia has shown acute lung injury. In the later stage of disease, patients can develop Acute Respiratory Distress Syndrome (ARDS) or ARDS-like conditions and multi-organ failure. Because of the sudden emergence of COVID-19, rapid research efforts are being conducted to repurpose existing approved drugs or biologic immunotherapies in this new indication, as these options are more likely to have near-term benefit during the pandemic. A major goal of many of these initiatives is to prevent or reduce the cytokine storm in pulmonary tissue. Animal model studies are informative at this juncture. SARS-CoVinfected mice have shown that $\mathrm{T}$-cell responses are required for protection from disease and for virus clearance. The immunomodulation by histamine depends mostly on its influence of T-cells. Histamine stimulates inflammation, cytokine release, and can lead to tissue damage, including lung. The immune system has innumerable ways to keep away viral invaders and keep them from returning. It selects for the B cells that produce antibodies capable of binding to the virus. It also squirrels away a store of long-lived memory B cells that produce those antibodies and that jump into action if the virus comes back. Another defense enlists $\mathrm{T}$ cells, which patrol the body seeking out and destroying infected cells, to disrupt the virus's ability to replicate. These immune cells can also tolerate for years $[4,5]$.

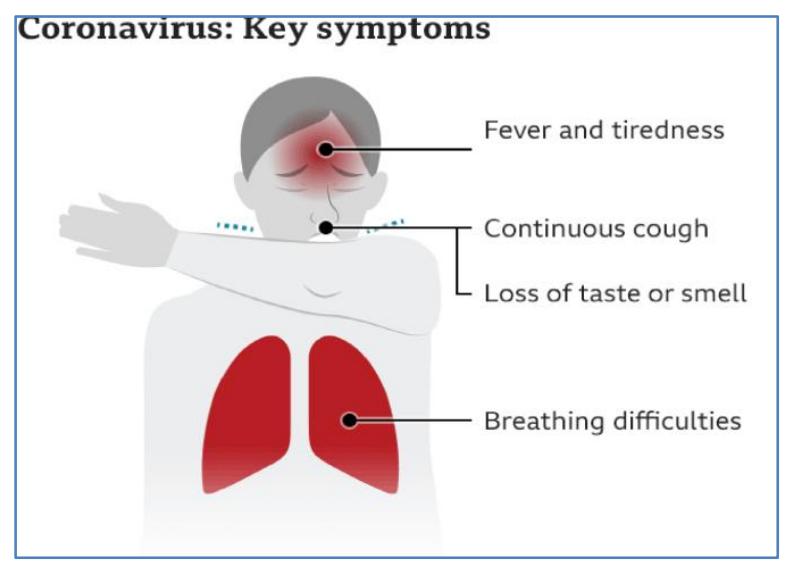

Fexofenadine is a selective, non-sedating $\mathrm{H} 1$ receptor antagonist, marketed in the United States since 2000. The FDA approved an oral suspension in 2006, for the treatment of seasonal allergic rhinitis and chronic idiopathic urticaria in children [6].

\begin{tabular}{|l|l|l|}
\hline S. No. & \multicolumn{2}{|c|}{ Description of Fexofenadine [7] } \\
\hline 1. & IUPAC & $\begin{array}{l}\text { 2-(4-(1-Hydroxy-4-(4 (hydroxydiphenylmethyl)piperidin-1-yl)butyl)phenyl)-2- } \\
\text { methylpropanoic acid. }\end{array}$ \\
\hline 2. & Average weight & $501.7 \mathrm{~g} / \mathrm{mol}^{-}$ \\
\hline 3. & Chemical Formula & $\mathrm{C}_{32} \mathrm{H}_{39} \mathrm{NO}_{4}$ \\
\hline 4. & Category & Histamine (H1) receptor antagonist \\
\hline 5. & Mechanism of action & $\begin{array}{l}\text { Fexofenadine is a highly selective and reversible competitor at peripheral H1 } \\
\text { histamine receptors in the gastrointestinal (GI) tract, blood vessels, and } \\
\text { bronchial smooth muscle. This agent interferes with mediators release from } \\
\text { mast cells either by inhibiting calcium ion influx across mast cell/basophil } \\
\text { plasma membrane or by inhibiting intracellular calcium ion release within the } \\
\text { cells. In addition fexofenadine may also inhibit the late-phase allergic reaction } \\
\text { by acting on leukotrienes or prostaglandins, or by producing an anti-platelet } \\
\text { activating factor effect. }\end{array}$ \\
\hline
\end{tabular}

\section{Materials ANd Methods \\ Molecular docking studies \\ Ligand Preparation}

2D Structure of ligand (FEXOFENADINE)

was drawn using Chem Sketch [8], the two-dimensional structure of was converted into 3-D structure and optimized with 3D geometry. The optimized structure was saved in PDB format for Auto-Dock compatibility. The basic structure of ligand (Fexofenadine) is given<smiles>CC(C)(C(=O)O)c1ccc(C(O)CCCN2CCC(C(O)(c3ccccc3)c3ccccc3)CC2)cc1</smiles>

2D structure of Fexofenadine below: 


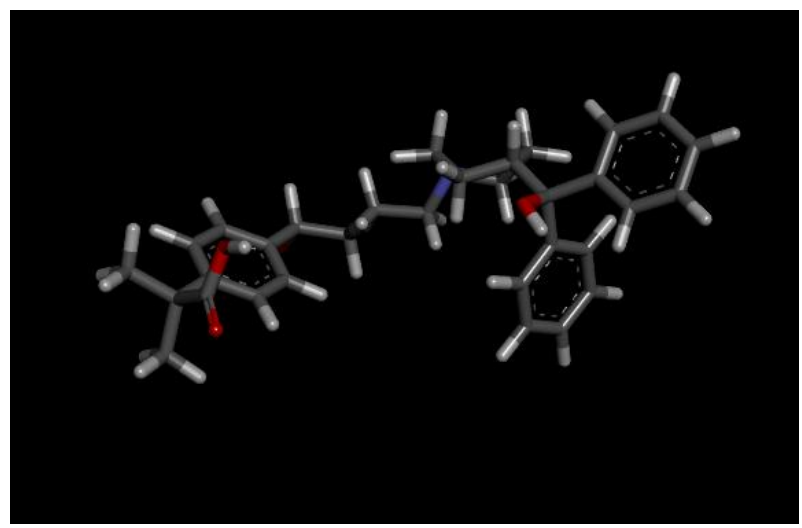

Fig-1: 2D and 3D conformer of Fexofenadine

\section{Preparation of the grid file}

The regions of interest used by Autodock were defined by considering grid area by making a grid box around the active sites. Grid box plays a central role in process of docking as it is made to cover all the amino acids present in active sites necessary for binding other than those present in receptor. Grid box has 3 thumbwheel widgets which let us change the number of points in the $\mathrm{x}, \mathrm{y}$ and $\mathrm{z}$ dimensions. The spacing between grids points can be adjusted with another thumbwheel, the value in the study taken is $0.419 \AA$ and No. of points considered are 40,54 and 40 points in the $\mathrm{x}, \mathrm{y}$, and $\mathrm{z}$ dimensions and $-9.732,11.403$ and68.925 as $\mathrm{x}, \mathrm{y}, \mathrm{z}$ centers $[9,10]$.

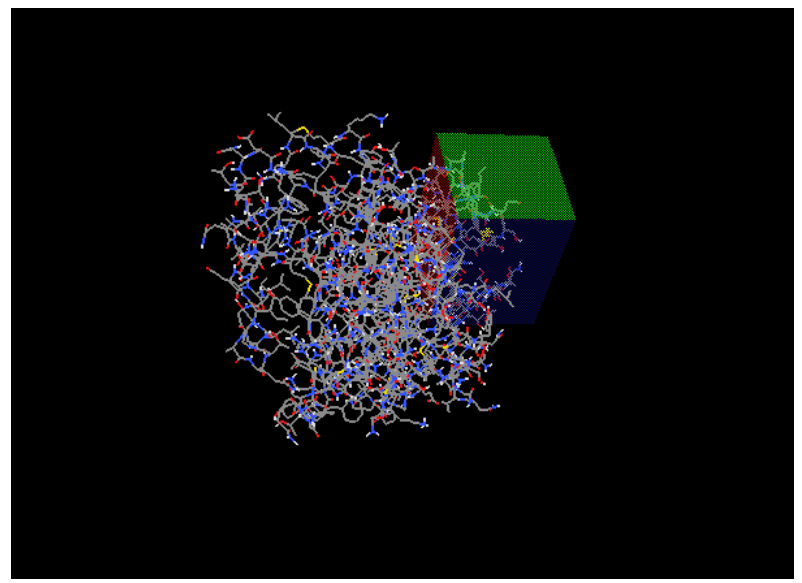

Fig-2: Grid box covering all active sites in receptor

\section{Preparation of the docking file}

All the calculations were carried out by using Autodock4.2 as docking tool. The visualization and other programs necessary for docking studies were performed out by means of Pymol, Chimera, DS visualizer, MMP Plus [11, 12].

\section{Docking of Main Protease with Fexofenadine Crystal structure}

The crystal structure of the protein consisting of receptor associated with bound ligand is downloaded from the Protein Data Bank portal. All the primary information regarding receptor and structure (6LU7.pdb) registered in the Protein data bank was used. The bound ligand peptide like inhibitor is found within the receptor [13].

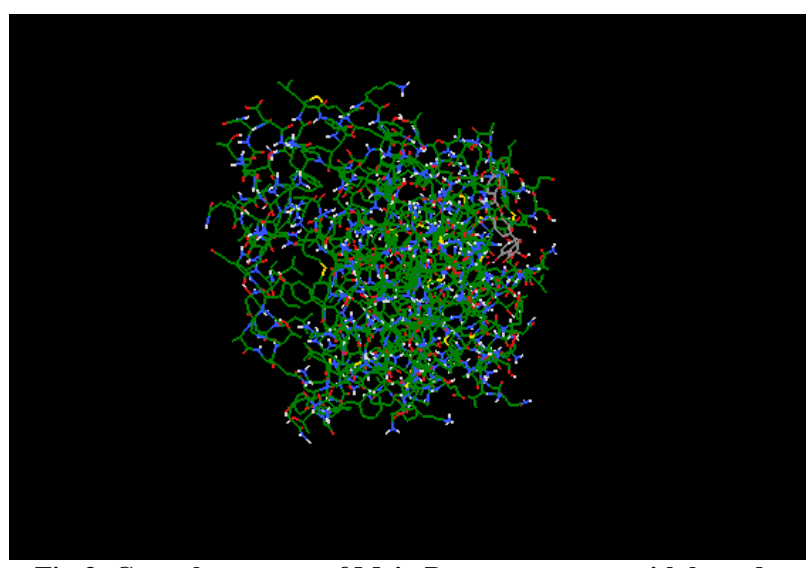

Fig-3: Crystal structure of Main Protease enzyme with bound peptide like inhibitor ligand (PDB ID-6LU7)

\section{Processing of Protein}

The downloaded receptor protein is having two chains $\mathrm{A}$ and $\mathrm{C}$, and both the chains have been used for experimental purpose. The bound ligand peptide like inhibitor was separated from the macromolecular complex by using software Chimera [14].

\section{Molecular Docking Simulation Studies}

Docking of Fexofenadine ligand on Main Protease enzyme was performed by Autodock. All the bonds of ligand were kept flexible, while no residues in receptor were made flexible [15].

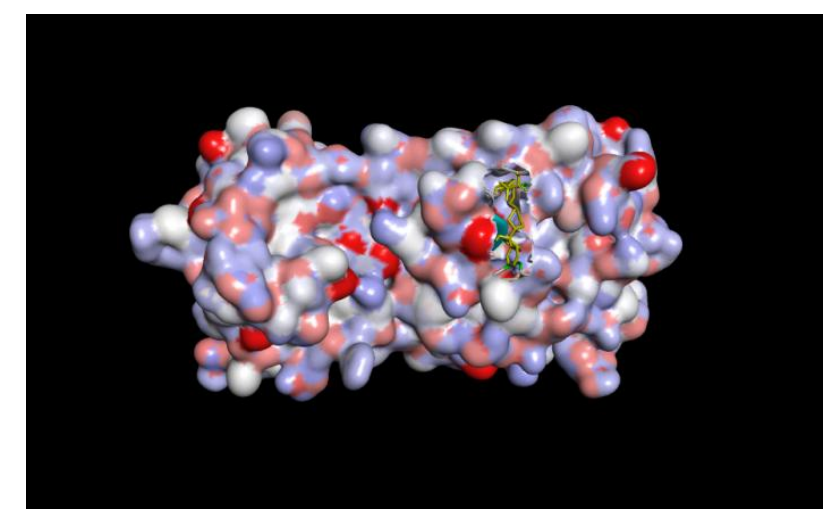

Fig-4: Binding mode of Fexofenadine within the active site of main protease Receptor

\section{Toxicity \& ADME-T Studies}

The modified lead molecules are studied by online program OSIRIS, for prediction of presence of any toxic group as well as presence of any toxic group and ADME- T properties [16].

\section{Results AND Discussion \\ Docking}

Following result were observed in docking studies of Main Protease enzyme with Fexofenadine. 
Table-1: Result of docking of Fexofenadine against Main Protease enzyme

\begin{tabular}{|c|c|c|c|c|c|}
\hline $\begin{array}{c}\text { S. } \\
\text { No }\end{array}$ & Compound Name & $\begin{array}{c}\text { Sinding Energy } \\
\text { (Kcal/mole) }\end{array}$ & Ki $(\boldsymbol{\mu M})$ \\
\hline 1 & FEXOFENADINE & & \\
\hline
\end{tabular}

The Fexofenadine was docked and the binding energy was found to be $-5.57 \mathrm{kcal} / \mathrm{mol}$.

\section{Interactions}

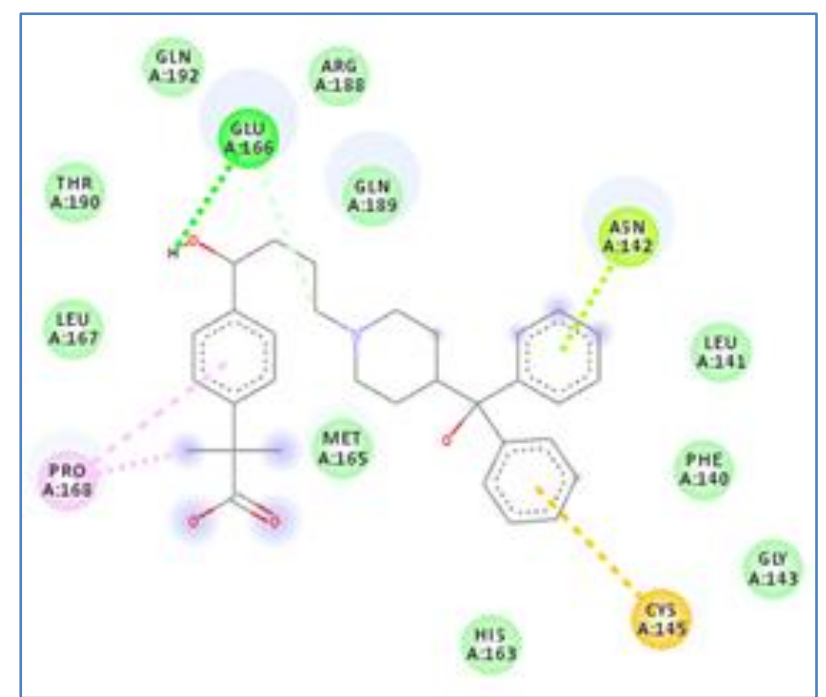

Fig-5: Binding interaction of FEXOFENADINE with main protease.

The Fexofenadine interacts with the Asn142, Cys145, Glu166, and Pro168 residues of main protease to form a complex structure.
Toxicity \& ADME-T Studies

The pharmacokinetic profile of FEXOFENADINE reveals that it is having good pharmacokinetic profile without presence of any major toxic effects. The pharmacokinetic and toxicity profiling results of FEXOFENADINE were shown in figure 2 .

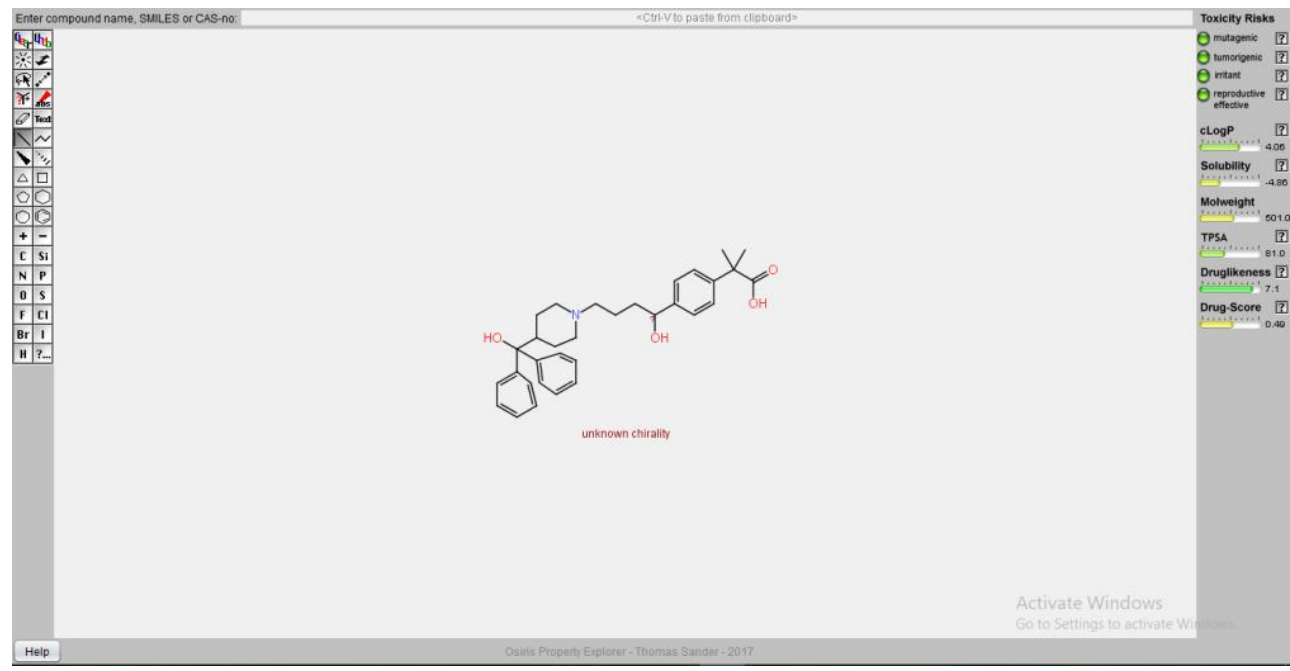

Fig-5: Pharmacokinetic and toxicity profiling of Fexofenadine. 
The molecular docking of FEXOFENADINE with Main Protease enzyme revealed that (Table 1), it has exhibited the chemical interaction with the amino acids in the active pockets which is showed in Figure.3. Theoretically, the ligand molecule has shown encouraging docking score. The docking result of FEXOFENADINE revealed that their docking scores was-5.57 kcal mol ${ }^{-1}$, and it can predicted as a very good inhibitor of Main Protease enzyme.

\section{Conclusion}

In modern era, weak immune response has urbanized due to random use of existing antimicrobial drugs in the treatment of infectious diseases. The consequential showed the outbreak of worldwide COVID-19(novel corona) having an exponential growth rate of a $16 \%$ increase in cases per day is shown. Approximately at present in India 3,546,705 active cases was identified and an above 63,690 deaths occurred. However preventive measures need to be done for spreading the SARs. From the present molecular docking investigation, it is concluded that Fexofenadine acts as a potent inhibitor of Covid-19 main protease and may act as a preventive drug for the treatment of SARS as it shows good binding affinity with the macromolecule with extremely good dock score and various binding interactions.

\section{REFERENCES}

1. Sharma, S. Corona: A review on current clinical sympathetic. Sch. J. App. Med. Sci,8(3), 10541061.

2. Malik, J. K., Kumar, A., \& Soni, H. Epidemiology of Novel Corona Virus (Covid-19): A Review.

3. Soni, H., Sharma, S., \& Malik, J. K. (2020). Synergistic Prophylaxis on COVID-19 by Nature Golden Heart (Piper betle) \& Swarna Bhasma. Asian Journal of Research in Dermatological Science, 21-27.

4. Malik, J. K., Soni, H., Sharma, S., \& Sarankar, S. Hydroxychloroquine as Potent Inhibitor of COVID-19 Main Protease: Grid Based Docking Approach.

5. Soni, H., Gautam, V. K., Sharma, S., \& Malik, J. K. Rifampicin as Potent Inhibitor of COVID-19 Main Protease: In-Silico Docking Approach.
6. Axelrod, D., \& Bielory, L. (2008). Fexofenadine hydrochloride in the treatment of allergic disease: a review. Journal of Asthma and Allergy, 1, 19.

7. https://pubchem.ncbi.nlm.nih.gov/compound/Fexof enadine.

8. ACD/Structure Elucidator, version 2018.1, Advanced Chemistry Development, Inc., Toronto, ON, Canada, www.acdlabs.com, 2019.

9. Morris, G. M., Huey, R., Lindstrom, W., Sanner, M. F., Belew, R. K., Goodsell, D. S., \& Olson, A. J. (2009). AutoDock4 and AutoDockTools4: Automated docking with selective receptor flexibility. Journal of computational chemistry, 30(16), 2785-2791.

10. Mujwar, S., \& Pardasani, K. R. (2015). Prediction of Riboswitch as a potential drug target for infectious diseases: An Insilico case study of anthrax. Journal of Medical Imaging and Health Informatics, 5(1), 7-16.

11. Mujwar, S., \& Pardasani, K. R. (2015). Prediction of riboswitch as a potential drug target and design of its optimal inhibitors for Mycobacterium tuberculosis. International Journal of Computational Biology and Drug Design, 8(4), 326-347.

12. DeLano, W. L. (2002). Pymol: An open-source molecular graphics tool. CCP4 Newsletter on protein crystallography, 40(1), 82-92.

13. Berman, H.M., Westbrook, J., Feng, Z. (2000). The Protein Data Bank. Nucleic Acids Res. Jan 1, 28(1):235-242.

14. Shah, K., Mujwar, S., Gupta, J. K., Shrivastava, S. K., \& Mishra, P. (2019). Molecular Docking and In Silico Cogitation Validate Mefenamic Acid Prodrugs as Human Cyclooxygenase-2 Inhibitor. Assay and drug development technologies, 17(6), 285-291.

15. Sharma, K. K., Singh, B., Mujwar, S., \& Bisen, P. S. (2020). Molecular Docking Based Analysis to Elucidate the DNA Topoisomerase II $\beta$ as the Potential Target for the Ganoderic Acid; A Natural Therapeutic Agent in Cancer Therapy. Current Computer-Aided Drug Design, 16(2), 176-189.

16. Thomas, S. Idorsia Pharmaceuticals Ltd, Hegenheimermattweg 91, 4123 Allschwil, Switzerland, Email: thomas.sanderidorsia.com. 